\title{
Experience of Integrative Medicine in the Hospice Ward of the University Hospital San Martino in Genoa, Italy
}

\author{
Nicola Traverso ${ }^{1 *}$, Stefano Bartoldi ${ }^{2}$, Davide Ferrari ${ }^{3}$, Cristina Bazzan ${ }^{4}$ and Michele Gallucci ${ }^{5}$ \\ ${ }^{1}$ Associate Professor in Clinical Pathology, University of Genova, Italy \\ ${ }^{2}$ Thesis Student, Degree Course in Medicine and Surgery, University of Genova, Italy \\ ${ }^{3}$ Music Therapist, IRCCS University Hospital San Martino, Genova, Italy \\ ${ }^{4}$ Art Therapist, IRCCS University Hospital San Martino, Genova, Italy \\ ${ }^{5}$ Head Physician of the Hospice Ward, IRCCS University Hospital San Martino, Genova, Italy
}

*Corresponding author: Nicola Traverso, Associate Professor in Clinical Pathology,

University of Genoa, Italy.

Received Date: December 30, 2020

Published Date: February 11, 2021

\section{Introduction}

During the last two years or so, I (NT) have been allowed to attend, as a tutor to a thesis medical student (SB), the Hospice Ward of IRCCS University Hospital San Martino, Genoa, Italy. The IRCCS Hospital San Martino is associated with the School of MedicalPharmaceutical Sciences (ex-Faculty of Medicine) of the University of Genova, Italy.

In this Ward, the Head physician, Prof. Michele Gallucci, has introduced the possibility of carrying out integrative medicine interventions, and in particular

$$
\begin{aligned}
& \text { - massage therapy } \\
& \text { - art therapy } \\
& \text { - music therapy } \\
& \text { - meditation } \\
& \text { - writing short stories. }
\end{aligned}
$$

The patients admitted to this ward are essentially patients with advanced stage cancers, for which the only useful therapies are considered to be the palliative ones, since the therapies against cancer would cause more harm than good. The hospitalization of such patients usually lasts for a maximum of a few weeks; many

of them end their lives in the ward, while others prefer to return home before death.

The ward environment is absolutely different from a typical hospital ward, and it is very reminiscent of a family environment. Each patient has his/her own private room, which is not numbered, but named after Streets of the historical center of Genoa. There is also a large terrace available for patients and their relatives, and a lounge where all patients who wish and feel can go, and where tea is served in the afternoon. In the lounge there is also an upright piano, which can be played by volunteers, of course in agreement with the medical and nursing staff, who supervise the lounge as well as all the other rooms.

\section{Use and aims of Integrative Medicine in the Hospice Ward}

Some years ago, Professor Gallucci decided to introduce Integrative Medicines, whose aim is not to replace conventional therapies, which are used in any case to alleviate the symptoms and physical suffering of patients: Gallucci's opinion is that the available drugs are sufficiently effective in this sense. The introduction of integrative therapies was specifically designed to evaluate their possible effectiveness on the patient's quality of life, on his/her 
acceptance of the disease, on the self-awareness of the near end of life and to observe whether these therapies are able to allow patients to fill with more intense meaning their last weeks of life. More specifically, reporting the words of the research protocol: "The patient, thanks to integrative therapies, explores his/her own condition and expresses his/her life experiences, becomes more aware of his/her own body and its complex balances. The general objectives are to improve the quality of life and well-being of patients, reducing the divergence between their expectations and what is really happening; to give shape to one's existence and continue to build one's life plan".

\section{Organization of work and Integrative Medicine in the Ward}

The ward holds plenary staff meetings about weekly, which my medical student and I have occasionally been able to attend. In these meetings, the head physician professor Gallucci plays the role of coordinator and stimulator of the discussion, but he is "primus inter pares" with respect to all the other participants, whether they are doctors, nurses, doctors in training, integrative therapists or guests.

The objective of the meetings is to make a point not only on the clinical progress of patients and the situation in the ward, but also to discuss the Integrative Medicine interventions that were carried out during that week.

It must be remembered that not all patients are eligible for Integrative Therapy, and sometimes even some eligible patients do not accept the intervention of the integrative therapist. The patient's specific sensitivity to each of the proposed integrative therapies must also be taken into consideration.

\section{Opinion of the Staff on Integrative Medicine}

Even though the number of patients accepted is small (the ward welcomes about ten patients at a time), and therefore it is difficult to obtain significant statistics, the members of the staff meetings claim to observe a positive effect of integrative therapies on patients; this effect can be different from patient to patient, and can consist in a better compliance with the therapies that the medical and health staff prescribe or administer to them, in an improvement of the acceptance of their condition, or even in a better acceptance of the conscience that life is coming to its end. A further often observed benefit is that the patient establishes a less medicalized relationship with the integrative therapist and is more inclined to reveal to the integrative therapist aspects of him/herself that seem to be more difficult to reveal to medical or healthcare staff.

\section{Some Interesting Cases and Possible Interpretation}

Personally, I have observed some cases that I considered of particular interest.

a) A patient followed the Story Composition Therapy and wrote a short story, which represented for him the awareness of having left a last sign of his existence. This experience made it possible for him to face death in a more serene way, with the conviction that he could continue to live beyond his death through the words of this story.

b) During a Music Therapy session, another patient evoked an image of calm waves gently crashing on a sandy shore. The patient also gave a title to this image, as if it were a painting: "Spring".

I believe that the evoked images can be comparable to unconscious images that can emerge during psychotherapy sessions. Here, we are faced with a limbo, a border between land and sea, which could be the representation of the border between life and death. But it can also be the representation of the moment of birth, with the detachment from the amniotic fluid, represented by the gentle water waves. We could therefore suggest that the patient evokes her close death as a possible rebirth, in an environment of calm and tranquility, and therefore we could perhaps conclude that the patient is able to visualize her near future, unfortunately inauspicious, with relative peace of mind.

It can also be noted that in this case, art therapy and music therapy interact.

c) A third interesting case was that of a patient who, during a Music Therapy session, evoked the image of a village on which the snow fell gently; she looked up and saw that it was the full moon literally snowing from the sky.

It is obviously a surreal image, and therefore most likely evoked from the unconscious of the patient. It is night, but the moon is full, and we know that this can give a peaceful brightness to the world, sometimes clearer and more significant than blinding sunlight. The Moon, perhaps a celestial mirror of the Great Mother Earth, dispenses a cloud of tranquility, a blanket of serenity on the village below, which awaits its destiny.

Also, in this case, we can sense the patient's ability or need to give a meaning of peace and calm to her destiny. In the embrace of the Great Mother, the patient seems to feel a security that will probably help her in the moment of her death.

\section{Conclusion and Limitations}

Of course, it should be noted that each interpretation would require longer times and numerous sessions to be confirmed, but, unfortunately, in the conditions of the patients of the Hospice, this possibility does not exist, due to the terminal condition of the patients.

Therefore, one of the objectives of Integrative Medicine activities could be that of approximating the goals that psychotherapy could achieve in much longer periods of time, unfortunately not available for this kind of patients. 
So, at least sometimes, Integrative Medicine could open shortcuts to reach the depth of the patients' unconscious and possibly resolve or at least attenuate anxieties and conflicts of these patients in a much shorter time than classic psychotherapy would do, and accompany them to their destiny, providing them with a greater sense of resignation, acceptance and peace.

\section{Acknowledgement}

I would like to sincerely thank all the Staff of the Hospice Ward, who actively participate in the management of the Hospice department with such a commendable commitment.

\section{Conflict of Interest}

Author declares no conflict of interest. 